\title{
Jean-Luc Marion: ¿fenomenólogo?
}

\author{
Azul Katz \\ Universidad de Buenos Aires/CONICET/Universidad de París IV-Sorbonne
}

Resumen: ¿Cómo entender la contradicción de una filosofia que, a la vez que se autoproclama heredera legitima de la fenomenología, pretende liberarse de sus límites y abrir paso a la manifestación incondicionada? ¿Debemos sin más leer la obra de Jean-Luc Marion como una verdadera renovación de la fenomenología, tal como él y sus seguidores pretenden o, por el contrario, debemos leerla como pura teología independizada del movimiento fenomenológico? ¿Por qué sería la vía teológica la única salida posible de la crisis de la metafísica contemporánea denunciada por Marion y, más aún, una vía necesaria? Para responder a estas y otras preguntas, el presente trabajo desarrolla algunas de las paradojas que surgen del giro teológico marioniano, distinguiendo dos maneras posibles de entender su Kehre: o bien como una desviación que permite extender la fenomenología a nuevos campos, o bien como un regreso o vuelta atrás y abandono del proyecto fenomenológico. Por último, nos preguntamos si la ontología donadora gradual, propuesta por Marion, no permite pensar en una posible tercera vía, según la cual el pensamiento de Marion no sería ya una fenomenología, sino una fansiología teológico gradualista.

Palabras clave: Jean-Luc Marion; giro teológico; fenomenología; intuición; donación

\begin{abstract}
Jean-Luc Marion: Phenomenologist?". How are we to understand the contradiction of a philosophy that claims to be a legitimate heir of the phenomenological tradition but, at the same time, intends to break free from its limits to allow an unconditioned manifestation? Should we comprehend Jean-Luc Marion's work as a true renovation of phenomenology -as he and his followers do-, or should we instead consider it as a pure theology independent from the phenomenological movement? Why would the theological way be the only possible way out of the crisis of contemporary metaphysics denounced by Marion and, even more, a necessary way? To answer these and other questions, this paper develops some of the paradoxes that arise from the Marionian theological turn, distinguishing two possible ways of understanding its Kehre: either as a deviation that allows extending phenomenology to new fields, or as a return to and abandonment of the phenomenological project. Finally, we wonder if Marion's proposal of a gradual ontology does not encourage a third possible way, which would lead us to think of his thought not as phenomenology anymore, but as a gradualist-theological fansiology.
\end{abstract}

Keywords: Jean-Luc Marion; theological turn; phenomenology; intuition; giveness 


\section{Introducción}

En 1990 apareció el ya célebre texto de Dominique Janicaud Le tournant théologique de la phénomenologie française ${ }^{1}$, con el cual se echaba luz, casi a modo de advertencia o denuncia, sobre el desvio teológico que había tomado la filosofia francesa de cuño fenomenológico. Por contraposición, Janicaud abogaba allí por una orientación minimalista de la fenomenología, es decir, por un fenomenología atea respetuosa de la reducción (o de la neutralidad del fenomenólogo) y de la inmanencia de la fenomenalidad. Probablemente, con el mismo gesto de separar la filosofía de la teología, Janicaud obligó a muchos a involucrarse en el debate y, por lo mismo, a tomar partido, de modo que cabe preguntarse si él mismo no terminó quizás contribuyendo a la consolidación de ese giro teológico.

Tal vez sea este también el caso de Jean-Luc Marion, cuyo interés por la teología, aunque presente desde sus primeras obras, se afirmó con la crítica a la onto-teología como modo de superar el "fin de la metafisica". La teología sería, en la obra de Marion, la vía alternativa a aquella filosofia que él critica. Ahora bien, incluso si aceptamos la crisis de la onto-teología, ¿puede sostenerse que la vía teológica es la única salida posible de la crisis de la metafísica contemporánea y, más aun, una vía necesaria? ¿No es acaso la teología una de las formas más metafísicas del pensamiento y, por tanto, aquella de Marion, una estrategia de simple sustitución de una forma por otra? Concedamos que Marion no entiende por teología toda la teología, sino la teología de la revelación, ni habla de la filosofia en su totalidad, sino de la filosofía-metafísica. Sin embargo, ¿por qué la salida de la filosofia-metafisica debe ser en dirección a una teología de la revelación en vez de hacia una filosofia-no-metafisica?

No esperamos ir aquí hasta el fondo de la cuestión de si la teología de la revelación se funda o no -y en qué medida- en un sistema metafísico. Lo que aquí nos interesa es considerar hasta qué punto el giro teológico puede convivir con una filosofia fenomenológica y cuál es el limite a partir del cual una filosofia teológica se independiza del proyecto fenomenológico para pasar a ser pura teología. De ningún modo debe entenderse este desafio como un mero

\footnotetext{
1 Janicaud, D., "Le tournant théologique de la phénoménologie française", en: La phénomenologie dans tous ses états, París: Gallimard, 2009.
} 
intento de trazar barreras arbitrarias, estáticas y predefinidas, que delimiten un campo legítimo para la fenomenología. Sin embargo, dado que desde su surgimiento la fenomenología no ha pretendido convertirse en toda la filosofia, consideramos legítima la pregunta por la extensión que subyace a su concepto, plástico y dinámico, pero no por ello inapresable o camaleónico. Para dar cuenta de estas diferencias, trataremos de establecer dos sentidos en que es posible entender el "giro" teológico, uno de los cuales, en principio, dejaría a la teología de Marion dentro del campo de la fenomenología, mientras que el otro, por el contrario, alejaría a nuestro contemporáneo francés del proyecto filosófico que ha comenzado con Husserl y que subsiste transfigurado en múltiples especies.

\section{Quiebre y ensanchamiento o el giro como desviación}

El primer sentido del término "giro" que tomaremos aquí es el de un desvio o viraje hacia, en sintonía con la consideración de Marion del giro como desarrollo y extensión del campo fenomenológico en una nueva dirección. De esta manera, la posición de Marion en la polémica janicaudiana dicta que "jamás hubo un 'giro teológico de la fenomenologia', pues la fenomenología desde un comienzo trató sobre temas teológicos... no se trata de un 'giro', sino de un desarrollo y una extensión del campo de intervención de la fenomenología”2.

\section{1.a. Mimética como superación}

En el primer capítulo de Réduction et Donation, titulado "La percée et l'élargissement", Marion hace una lectura del quiebre que introdujeron las Investigaciones lógicas de Husserl, a partir de las interpretaciones de Heidegger y Derrida: mientras que para Derrida las Investigaciones no representan un verdadero quiebre, porque no serían más que otro momento en la historia de la metafísica de la presencia (dado que se considera fenómeno a aquello que se presenta, intuitivamente), para Heidegger lo que las Investigaciones lógicas vienen a quebrar es el límite de la donación intuitiva como donación sensible. El motivo es que, con la intuición categorial, Husserl ensancharía el alcance de la intuición hasta lo conceptual, es decir, hasta el significado o las ideas. La ruptura con la que entra en escena la fenomenología a la historia de la filosofia sería, entonces, la extensión del dominio de la intuición hasta incluir

2 Marion, J.-L., La rigueur des choses. Entretiens avec Dan Arbib, París: Flammarion, 2012, p. 205. Las cursivas son nuestras. Todas las traducciones son nuestras directas de los originales franceses (o ingleses), excepto indicación contraria. 
-en términos de la Crítica de la razón pura- las categorias del entendimiento. Independientemente de la fidelidad o exactitud de dichas interpretaciones, esta lectura cumple una función clara en Marion: replicando el gesto husserliano de quiebre y ensanchamiento de la "fenomenología" kantiana -como diría Ricoeur3-, el autor francés legitima su propia propuesta como quiebre y ensanchamiento de la fenomenología husserliana. Si Husserl alcanzó un "ensanchamiento de los conceptos de intuición y percepción [hasta] la 'intuición categorial' como "intuición universal"', según Marion "los límites fijados por Kant a la intuición deben [todavia] transgredirse... por un solo motivo: la exigencia fenomenológica de la donación en presencia de todo fenómeno, sin resto ni reserva"s.

En realidad, esta "mimética metodológica"6 reitera, como dirá Dominique Janicaud, la mimética metodológica que utiliza Marion como estrategia para inscribirse, con su reducción pura a la donación, en la linea sucesoria de Husserl (reducción trascendental) y Heidegger (reducción existencial). El "quiebre y ensanchamiento" (según Réduction et donation) o el "desarrollo y ampliación" (según La rigueur des choses) que introduce Marion respecto al de las Investigaciones lógicas, como reducción a la donación, es el de la reconducción a aquello que es anterior a (y por tanto permite) la correlación (entre intención e intuición, entre nóesis y nóema, entre ego-cogito-cogitata) a la que se accede por medio de la reducción trascendental: "El quiebre fenomenológico no consiste ni en el ensanchamiento de la intuición, ni en la autonomía de la significación, sino en la primacía sola incondicionada de la donación del fenómeno... Intuición e intención no darían nada... si todo no debiera de entrada y en virtud del principio de la correlación ser dado para poder aparecer [estar dado para aparecer/être donné pour apparaître]"7.

\footnotetext{
3 Ricœur distingue entre una fenomenología kantiana (en tensión con una ontología), una fenomenología hegeliana (que desaparecerá en una ontología) y una fenomenologia propiamente husserliana que ni entra en tensión con la ontología ni marcha hacia una ontología, sino una fenomenología según la cual "no hay nada más en el ser o en los entes que aquello que aparece al hombre y por el hombre" (Ricœur, P., "Sur la phénoménologie", en: A l'école de la phénomenologie, París: Vrin, 1986, p. 144).

3804 Marion, J.-L., Réduction et donation, París: PUF, 2015, p. 28. En adelante, citado como RD.

5 Marion, J.-L., RD, p. 28.

6 Janicaud, D., "Le tournant théologique de la phénoménologie française", p. 97. La cita dicta: "Tenemos aquí la confesión de esta mimética metodológica por la cual se quiere hacer pasar el salto mortal hacia el 'llamado puro' como procedente del mismo rigor que el profundizamiento heideggeriano de la reducción husserliana".

7 Marion, J.-L., RD, pp. 58-59. Las cursivas son nuestras. Quizás sea forzar una obviedad, como es el hecho de que en español être puede traducirse por "ser" o por "estar", pero en este caso creemos que vale la pena usufructuar de la diferencia entre una y otra traducción ya que, a nuestro entender, "estar dado" no indica necesariamente a un agente, mientras que "ser dado"
} 
Lo que Marion espera introducir como novedad respecto de sus antecesores fenomenólogos es la apertura a la donación pura, hacia una donación "absoluta, libre y sin condiciones"8, y esta "apertura a la donación pura" se logra por reducción. "A mayor reducción, mayor donación" quiere decir, en este contexto, principalmente una reducción (como un dejar-fuera-de-juego) de las condiciones de posibilidad impuestas a la posibilidad de aparecer, esto es, a la fenomenalidad de los fenómenos: "Los fenómenos que no aparecen más que bajo condiciones, [son] alienados según una fenomenalidad impuesta"10. Son estos límites -estas condiciones para lo que puede darse en el modo de lo fenoménico- los que deben ser quebrados y superados: " $\mathrm{El}$ ensanchamiento debe entenderse no solo como una extensión (Erweiterung) de la intuición y de la significación por la donación, sino sobre todo como 'la exigencia de una liberación del suelo (Freilegung des Bodens) fenomenológico'. La donación ensancha la presencia porque la libera de todos los límites de las facultades, hasta dejar jugar libremente el ente -eventualmente el ente en su ser. Y solo un ensanchamiento así liberador podrá pretender sobrepasar la 'metafisica de la presencia' que, de hecho, no cesa de restringir el presente y de retener la donación"11.

Ahora bien, ¿a qué se refiere precisamente nuestro autor con estos límites de los que debe liberarse la fenomenalidad para poder manifestarse incondicionalmente? Pues bien, son los límites que los metafísicos de la presencia y los fenomenólogos de la modernidad le han impuesto. La principal restricción que el metafísico impone al fenómeno es la del límite entre lo posible y lo imposible. Kant representa un caso ejemplar de este proceder ya que, por temor a lo infinito e imposible, le impone a la fenomenalidad, como venimos de citar, "los limites de las facultades". En otras palabras, impone a priori las condiciones de posibilidad de todo aparecer fenoménico al definir el fenómeno como aquello que se adecúa a las condiciones de toda experiencia posible, es decir, a la intuición y los conceptos como las dos únicas fuentes del conocimiento. Cuando Kant se pregunta qué ocurriría con el fenómeno si no se acordara y correspondiera con las facultades de conocer del sujeto, responde que "un tal

pareciera requerir un actor. "Estar dado", por lo tanto, pareciera permanecer dentro del campo fenomenológico, mientras que "ser dado" anticipa la dirección hacia la cual nos quiere llevar Jean-Luc Marion.

8 Ibid. Las cursivas son nuestras.

9 Ibid.

${ }^{10}$ Marion, J.-L., Étant donné, París: PUF, 2013, p. 19. En adelante, citado como ED.

${ }^{11}$ Marion, J.-L., RD, pp. 67-68 
fenómeno simplemente no aparecería; o más bien no habría fenómeno en absoluto, sino una confusa aberración perceptiva sin objeto"12. Así, queda excluida para el conocimiento la posibilidad de una adecuación entre los fenómenos y las condiciones propias de la experiencia del sujeto trascendental. Sin embargo, ante todo, lo que Kant prohíbe, subraya Marion, son otros modos de intuición aparte de la sensible, es decir, la intuición intelectual. Es de esta prohibición de la que Marion extrae un privilegio de la intuición sobre el concepto, justamente, porque es la intuición la que da (más allá de que intuiciones sin conceptos sean ciegas). Así, "en el reino del fenómeno", nos dice Marion, "el concepto no es el rey, sino más bien la intuición ya que sola dispone del privilegio de donar... La fenomenalidad de la donación se indexa sobre la intuición"13.

Por su parte, si bien según Marion (y también según Heidegger) Husserl supera a Kant al postular la intuición categorial (de mayor alcance que la sensible), esta también es interpretada como una limitación por temor a la fenomenalidad. Marion sostiene que "Husserl casi llega [a la donación]. [Pero] sin dudas, él se contenta, (con muy poco costo), con nombrar esta donación, sin realmente pensarla. ¿Cómo la nombra? Por la finalmente muy limitada analogía con una intuición categorial, que, con un nuevo (o más bien muy viejo) sintagma, disimula el abismo de donación del ser... El quiebre [de las Investigaciones] va hasta la donación del ser, pero retrocede delante de su abismo, encerrándolo por el concepto... Heidegger, al contrario de Husserl, se dedicará a pensar la donación, y por tanto destruirá la intuición categorial -y de hecho, no la evocará más ni en Sein und Zeit ni después-"14.

Pero no es solo con la intuición categorial que Husserl limita la fenomenalidad: en Étant donné, Marion toma como punto de partida los principios fundamentales de la fenomenología de Husser ${ }^{15}$ para mostrar las limitaciones esenciales que se ejercen sobre la fenomenalidad. En primer lugar, el horizonte (de indeterminación determinable) co-dado con el fenómeno exhibe la inadecuación esencial entre lo mentado y lo dado; la experiencia es siempre perfectible y por tanto, para Marion, Husserl tomaría como modelo de fenomenalidad el

\footnotetext{
12 Marion, J.-L., ED, p. 350.

13 Marion, J.-L., ED, pp. 318-319. Las cursivas son nuestras.

14 Marion, J.-L., RD, p. 66. Las cursivas son nuestras.

15 Principio según el cual "toda intuición en que se da algo originariamente es un fundamento de derecho del conocimiento; que todo lo que se nos brinda originariamente (por decir asi, en su realidad corpórea) en la 'intuición', hay que tomarlo simplemente como se da, pero también solo dentro de los limites en que se da" (Husserl, E., Ideas relativas a una fenomenología pura y una filosofia fenomenológica, Gaos, J. (trad.), México/Buenos Aires: FCE, 1986, p. 58, §24).
} 
de los fenómenos deficientes en intuición (deficientes en el sentido de que la mayoría de las veces no se dan de manera adecuada, excepto cuando se trata de fenómenos abstractos o matemáticos). El paradigma matemático de evidencia resulta, por lo tanto, deficiente para el modelo de fenomenalidad que Marion busca justificar. En segundo lugar, el yo trascendental, se argumenta, es erróneamente considerado como constituyente ya que, de hecho, es al exponerse a la donación que deviene constituido. De este modo, Marion opera una inversión de la relación de constitución dejando al sujeto en el lugar de testigo de la donación: su función es pasiva, es la de atestiguar cómo se da la donación a través de los fenómenos.

Ahora bien, para Marion, la manera de salir de la limitación metafisica es pensando grados de donación. En lugar de determinar las condiciones de la experiencia o el principio de todos los principios a los cuales debe adecuarse todo fenómeno para poder ser tal, Marion propone eliminar la limitación impuesta para dejar que el fenómeno se muestre a sí mismo en distintas medidas. De hecho, quizás sorprenda que pese a su crítica del principio de todos los principios, Marion sostenga que mantendrá "en todos los casos la definición genérica [de fenómeno] como aquello que se muestra a partir de sí (Heidegger)"16. En definitiva, aquello que busca introducir Marion, además del desplazamiento hacia la donación (¿y por qué no, preguntémonos, hacia el polo objetivo de la correlación husserliana?), es la gradación en la donación como modo de escapar a la regionalización metafisica de la fenomenalidad.

\section{1.b. Variaciones fenoménicas o los grados del aparecer}

Ahora bien, si aceptamos, con Marion, que hay una gradación o degradación ontológica donadora -esto es, de la donación que se modaliza en las diversas figuras de la fenomenalidad-, ¿no deberiamos también preguntarnos por los extremos de esa modalización? Dicho de otro modo, ¿no oscilaría la fenomenalidad entre un mínimo y un máximo de mostración o saturación que le corresponden propiamente sin venirle impuestos desde fuera?

En los libros III y IV de Étant donné, Marion comienza considerando la donación en general como aquello que rige universalmente la fenomenalidad, como aquella condición última de posibilidad de todo lo que aparece. Podriamos incluso pensar que la donación es a los fenómenos lo que el ser a los entes,

16 Marion, J.-L., ED, p. 364. 
dado que es ella quien da y "nada [la] precede"17. Así, la donación "se articula en todos los modos del fenómeno, cualesquiera que sean. Más 'ensanchada' que la intuición, más autónoma que la significación, la donación da el fenómeno a través de ella"18. De este modo se abre paso lo que podemos llamar una fansiología gradualista o de-gradación fenoménica, la cual da lugar al espectro de variación de los fenómenos en que esa donación se manifiesta ${ }^{19}$.

La diversidad de fenómenos en que la donación se modaliza se explica a partir de las variaciones que se dan en la relación entre intención e intuición. Dentro de esa diversidad, Marion distinguirá en principio tres tipos de fenómenos: (a) los fenómenos pobres, faltos de intuición, que tienen el privilegio de la certeza porque, en la medida en que son abstractos, no pueden ser decepcionados por una intuición; (b) los fenómenos de derecho común, en los cuales la adecuación es posible pero no se cumple; y (c) los fenómenos saturados, aquellos en los que la intuición siempre satura a la intención ${ }^{20}$. Esta saturabilidad de los fenómenos es el principal resultado de la reducción a la donación, porque la reducción permite la aparición no solo de lo más irreductible de los fenómenos (el hecho de que son dados), sino también la aparición de la versión más colmada del fenómeno: "A la posibilidad reducida de la fenomenalidad, ¿no debemos -en ciertos casos todavía por definir- oponer una fenomenalidad incondicionalmente posible y en la cual la medida no resultara de la finitud de las condiciones de la experiencia? Al fenómeno supuestamente pobre en intuición, ¿no podemos oponer un fenómeno saturado de intuición?”21. Es por

17 Marion, J.-L., RD, p. 60.

18 Ibid.

19 Utilizamos el término "fansiologia" como sustituto de "fenomenologia" pues permite ver el desplazamiento que habría de la fenomenología de Husserl a la filosofia teológica de Marion: aunque ambos términos contengan la raíz en "phaino" (aparecer), la "fenomenología" sería la ciencia de los fenómenos, mientras que la "fansiología" sería la ciencia del aparecer.

${ }^{20}$ Se pueden, en este punto, postular objeciones significativas a la idea de que, por ejemplo, en Husserl solo habría fenómenos pobres y fenómenos de derecho común pero no fenómenos saturados. Como nos recuerda el Dr. Walton en su artículo "Reducción fenomenológica y figuras de la excedencia”, en: Tópicos, 16 (2008), pp. 169-187, los fenómenos en Husserl son saturados, ya que, en lo que se muestra siempre hay referencias a lo que no está presente, es decir, siempre en ellos hay excedencia, por ejemplo, por los horizontes espacio-temporales que participan necesariamente en la constitución de cada objeto de la percepción.

21 Aclara Marion que el fenómeno es saturado y no saturante porque "la intuición que lo satura lo satura únicamente en nombre de la donación: el fenómeno saturado lo es ante todo de donación. Seguramente un fenómeno tal satura también y por consecuencia la mirada a la cual se dona para ver y conocer; en rigor por tanto también lo podemos llamar saturante. Por lo tanto, la saturación que ejerce en el campo del conocimiento resulta solamente de aquella que recibe en el campo de la donación: la donación determina siempre el conocimiento y no a la inversa" (Marion, J.-L., ED, p. 325, n. 1). 
ello que "Husserl, que se excedía de la metafisica kantiana del fenómeno, debe a su vez ser superado para acceder a la posibilidad del fenómeno saturado"22.

\section{1. c. Los fenómenos saturados: ¿regla o excepción?}

Marion ejemplifica lo que es un fenómeno saturado con casos de la historia de la filosofia como, por ejemplo, la idea de infinito en Descartes, la conciencia interna del tiempo en Husserl y la experiencia de lo sublime en Kant. El último ejemplo nos resulta más resonante ya que, de hecho, Marion caracteriza al fenómeno saturado según las cuatro categorías que conducen el análisis kantiano en la "Analítica de lo Bello" y la "Analítica de los sublime" de la Crítica del juicio. (1) Según la cantidad, el fenómeno saturado es invisable (in-mirable), no se puede mentar. (2) Según la cualidad, el fenómeno saturado es insoportable, vuelve imposible sostener la mirada porque la saturación produce un deslumbramiento similar, dirá Marion, al que afecta a los prisioneros de la caverna de Platón cuando salen por primera vez al mundo. (3) Según la relación, el fenómeno saturado es absoluto, no relativo, porque "se sustrae a toda analogía de la experiencia"23. Finalmente, (4) según la modalidad, el fenómeno saturado es irregardable, es decir, no se deja ver completamente ${ }^{24}$. ¿No podríamos entonces acaso insertar la siguiente cita de Marion en la Crítica del juicio de Kant para describir el fenómeno estético y, más específicamente, el de lo sublime?: "El fenómeno saturado se resiste a dejarse mirar como un objeto, precisamente porque aparece con un exceso múltiple e indescriptible que anula todo esfuerzo de constitución. Es necesario determinar el fenómeno saturado como un fenómeno no objetivo o más exactamente no objetivable"25.

\footnotetext{
${ }^{22}$ La cita es la conclusión de lo que sigue: "El principio de todos los principios menciona al horizonte y al yo constituyente como los presupuestos ellos mismos incuestionados de todo aquello que se constituye en general como fenómeno; mientras que el fenómeno saturado, en tanto que incondicionado por un horizonte e irreductible a un yo aspira a una posibilidad liberada de esas dos condiciones; y contradice por tanto el principio de todos los principios" (ibid., pp. 357-358). Las cursivas son nuestras).

${ }^{23}$ Ibid., p. 340.

${ }^{24}$ Insistimos en la concordancia entre los planteos de Kant y Marion para resaltar, entre otras cosas, la encrucijada a la que nos vemos conducidos por un planteo que primero condena la filosofia del límite de Kant, pero luego utiliza como ejemplo uno de los fenómenos más extraordinarios entre los analizados por Kant. De hecho, la no objetivabilidad del fenómeno saturado es similar a la no objetivabilidad del fenómeno estético kantiano y esta negatividad se refleja en las categorias con que los dos autores describen las experiencias que los convocan: para Marion: in-visable, in-supportable, $i$-rregardable, $i$-rrelativo/no-relativo/in-analogizable para lo absoluto; para Kant des-interés, universalidad subjetiva, finalidad sin fin, necesidad condicionada.

${ }_{25}$ Marion, J.-L., ED, p. 351. Asimismo otras frases podrian sustituirse en un texto y en otro, por ejemplo: "La finitud se descubre más delante del fenómeno saturado [lo sublime -matemático y
} 
Ahora bien, podriamos suponer que con "fenómeno saturado" Marion se refiere a casos extraordinarios, en el sentido de casos límite o de experiencias del límite y en el límite, como puede ser el ejemplo del problema husserliano de la conciencia interna del tiempo (la dificultad no se encuentra en el nivel de la objetivación o constitución regular de objetividades, sino cuando hay que dar cuenta de la constitución de la totalidad o unidad de la conciencia, es decir, en la manifestación del punto superior en el proceso de constitución de la conciencia misma ${ }^{26}$ ). Sin embargo, sorprende la insistencia en que el fenómeno saturado no es "un caso excepcional que mencionamos para recordar al margen del fenómeno pobre, supuestamente más frecuente y por tanto aproximativamente normativo [sino que] se trata, por el contrario, de una determinación esencial del fenómeno"27. Más aun, Marion propone una inversión en el orden de la fundamentación al sostener que "la metafísica piensa siempre el fenómeno de derecho común (penuria de intuición) a partir del fenómeno pobre en intuición. Toda nuestra tarea tiende al contrario a pensar el fenómeno de derecho común y, a través de él, el fenómeno pobre, a partir del paradigma del fenómeno saturado"28.

Postular la saturación como el máximo de donación, ¿no implicaría limitar nuevamente la fenomenalidad o, directamente, cambiarle el nombre a ese fenómeno límite? Dicho de otro modo, ¿puede Marion ser consecuente con su objeción ubicando el fenómeno saturado o pleno como modalización extrema de la donación? No, y es por ello que aquí comienzan las apuestas más arriesgadas de su planteo y, con ellas, las objeciones más serias: "No podemos evitar concluir que en el interior de la región privilegiada de la saturación, todos los fenómenos, todos saturados, no ofrecen el mismo grado de donación. La pregunta que se impone así es determinar hasta qué grado la saturación puede desplegarse, si ella alcanza un máximo o si, por hipótesis, ella los transgrede siempre -pero también si hay algún sentido en que se puede considerar un fenómeno que se dona 'luego' de un máximo de fenomenalidad”29.

dinámico-] que del fenómeno pobre [lo bello]" (ibid., p. 339).

${ }^{26}$ Por supuesto, dado que el problema atañe al estaño superior de la conciencia (cómo es constituida la conciencia constituyente), todos los estaños inferiores se ven afectados. Pero como problema metodológico es un problema en el límite de la fenomenología husserliana, porque, entre otras cosas, la dificultad tiene que ver con el ingreso en un espacio inefable o paralingüístico que oscurece profundamente, y por tanto dificulta hasta poner en jaque, la descripción fenomenológica.

${ }^{27}$ Marion, J.-L., ED, p. 339. Las cursivas son nuestras.

${ }^{28}$ Ibid., p. 372. Las cursivas son nuestras.

${ }^{29}$ Ibid., p. 383. 
Estas preguntas nos enfrentan a la última de las figuras de la donación: (d) el fenómeno doblemente saturado o, con el nombre que recibe en Étant donné, el fenómeno de la revelación. Pero, ¿̇acaso no significa tal postulación no una ampliación, sino solamente el quiebre, y por tanto el abandono, de la idea de fenomenología? ${ }^{30}$.

\section{El giro teológico como vuelta en $U$}

Un segundo sentido de "giro" sería el del retorno o "giro en U", entendido como un desandar el camino por el que nos hemos desviado, una vuelta atrás. Con esta metáfora automovilística apuntamos a señalar que identificar la filosofia-metafisica con la filosofia moderna lleva a Marion a concluir que para superar o escapar o desviar a la filosofia de sus pecados metafisicos, se debe volver a una era pre-metafisica o pre-moderna. "Volver a" significa cuanto menos volver a pensar despojándose o haciendo abstracción de los modos del pensamiento y los prejuicios que heredamos del pensamiento moderno, en busca de un destino diferente de aquel al cual esos prejuicios nos han conducido, es decir, la onto-teología. Esta vuelta atrás la encontramos en, al menos, dos indicaciones de Marion. La primera y más reciente es un llamado a volver a pensar a partir de San Agustín, releyéndolo "desde un punto de vista al menos negativamente identificable: un punto de vista no metafisico"31. Este llamado al punto de vista del pensamiento premoderno se emparenta, naturalmente, con aquella perspectiva a la que Marion aspira: "nuestro utópico contemporáneo... pensar desde un punto de vista postmetafísico" 32 .

La segunda indicación la encontramos en algunos de sus estudios estéticos. En "El cruce de lo visible y lo invisible"33, por ejemplo, Marion comienza mostrando cómo la perspectiva en pintura ejercita el reconocimiento de lo invisible como condición de aparición y visibilidad de lo visible, para concluir que es más bien con el icono que este procedimiento se exacerba, al dejar en

\footnotetext{
30 Aunque algunas de estas objeciones Marion las ha tratado en libros más recientes, como Le visible et le revelé, creemos que en esencia su postura de $E D$ no se ha modificado, sino intensificado. Prueba de ello es el artículo, del que nos ocupamos hacia el final del presente trabajo: Marion, J.-L., "On the Foundation of the Distinction Between Theology and Philosophy", en: Budhi: A Journal of Ideas and Culture, v. XIII, 1-3 (2009), pp. 55-56.

${ }^{31}$ Marion, J. L., Au lieu de soi. L'approche de saint Augustin, París: PUF, 2008, p. 28.

32 Ibid.

${ }^{33}$ Marion, J.-L., "El cruce lo visible y lo invisible", en: El cruce de lo visible, Castellón: Ellago Ediciones, 2006. En adelante CV.
} 
diálogo dos miradas invisibles que se entrecruzan. No obstante, ¿̇a qué se debe esta recuperación del icono?

Luego de destacar el privilegio de la pintura para manifestar la potencia de lo invisible en relación con lo visible, Marion nos recuerda que, así como la visión binocular introduce la profundidad "real" en el espacio de manera que podamos distinguir entre objetos, la perspectiva en el cuadro introduce una profundidad "virtual" o "ideal" ya que deja aparecer distintos planos en medio de un plano real único y llano (el del lienzo): "la mirada en perspectiva separa lo visible por la potencia constante de lo invisible de tal manera que nos lo hace vasto, habitable, organizado"34. Tanto la visión binocular como la perspectiva ponen en escena lo visible en un médium invisible, esto es, en un vacío. Solo que, mientras la mirada "real" lo hace con un vacío cósico o físico, la perspectiva en pintura lo hace con un vacío ideal e invisible que se revela como la condición de posibilidad de lo visible en la medida en que permite que aparezca, que se vuelva visible. Este vacío que introduce lo invisible en la imagen puede tomar distintas formas: por ejemplo, un espejo abre una puerta al infinito, pero naturalmente lo esencial en la perspectiva es el punto de fuga que señala la dirección de la infinitud. De hecho, no es que "la perspectiva [abra] ninguna nueva dimensión, sino que suprime una dimensión real (la profundidad en la que mis pasos podrian avanzar)"35. A modo de ejemplos, Marion toma de un lado al impresionismo (Monet) como un tipo de arte que se inclina hacia el polo subjetivo de la correlación fenomenológica, en la medida en que la experiencia se retrae en la función interpretativa o constituyente del sujeto, casi destruyendo la trascendencia del objeto intencional, para volverse "vivencia sin objeto". Del otro lado, como arte en que aparece un objeto sin vivencia, o un objeto que se autonomiza del sujeto, Marion propone al suprematismo (Malevitch).

Ahora bien, lo que pone en valor el "ver a través"36 que caracteriza a la perspectiva es la importancia de "lo invisible [que] desequilibra lo visible para obligar a la mirada a ponerse en movimiento y así, gracias a la inmovilidad del cuadro, a verlo" 37 . Dicho de otro modo, ese "ir más allá de lo dado" lleva en sí la necesidad de superar a su vez la perspectiva en busca de una invisibilidad superior. De modo que, a esa primera manera que tiene lo invisible de cruzarse con lo visible (en el impresionismo de Monet o en el suprematismo de Malevitch,

\footnotetext{
34 Marion, J.-L., CV, p. 22.

35 Ibid., p. 30.

36 Ibid., p. 33.

37 Ibid. p. 29.
} 
o también en la anamorfosis como exacerbación de la función de la perspectiva), Marion opone una segunda posibilidad en que lo invisible aparece: el icono.

El icono o la pintura icónica suele requerir no ya un ejercicio de la perspectiva (esto es, un juego de variación en la correlación espacial entre el cuerpo propio y el objeto visto), sino un juego que se libera de lo visto para introducirnos en un mundo invisible, más potente que el visible. El icono tiene, según Marion, tres características: primero, no moviliza la perspectiva; segundo, muestra siempre una mirada (una "mirada pintada [que] contesta invisiblemente la invisible mirada del orante y transfigura su propia visibilidad incluyéndola en el comercio de dos miradas invisibles -la mirada del hombre rezando-, a través del icono pintado, a la mirada santa invisible, y la mirada santa invisible recubriendo con benevolencia, a través del icono visiblemente pintado, la mirada del orante"38); tercero, invierte la relación no solo entre lo visible y lo invisible, sino también entre el espectador y el espectáculo, de manera que lo central pasa a ser lo invisible. No se trata, por lo tanto, de una mera superación de la perspectiva, sino más bien de una abstención de perspectiva: "en esta situación, no hemos salido de la perspectiva porque aún no hemos entrado en ella: lo invisible tiene algo mejor que hacer, en pintura, que poner en escena lo visible o incluso que denunciar esta puesta en escena objetiva" 39 . Ahora bien, esta suerte de neutralidad o unidimensionalidad del icono, que en términos de Husserl podría explicarse como una insuficiencia en la función de "apuntar a" de la imagen objeto hacia la imagen sujeto (en la conciencia de imagen) ${ }^{40}$, a nuestro entender encuentra un lugar primordial en la estética de Marion porque obliga a ver mucho más allá de lo visible. En verdad, el valor del icono en la filosofia de Marion resulta de ser un tipo de pintura preperspectivista y, por lo tanto, prerenacentista, precientificista; en conclusión, premoderna. Marion nos invita así, una vez más, a perder la perspectiva para entregarnos a un intercambio "litúrgico y oratorio" 41 : "Lo visible, que el pintor mismo, rezando, coloca sobre la madera, se despliega saturado por lo invisible de las miradas intercambiadas. Lo invisible ya no se consagra ni se pierde sirviendo a lo visible, como en la perspectiva... El icono se apoya pictóricamente en la inclusión, dentro de la mirada humana, de lo invisible en

\footnotetext{
38 Ibid. pp. 46-47.

39 Ibid. p. 47.

${ }^{40}$ Cf. Hua XXIII, especialmente el texto $\mathrm{N}^{\circ} 1$, o también el $§ 111$ de Ideas $I$.

${ }^{41}$ Marion, J.-L., CV, p. 46.
} 
el seno de lo visible para obrar así, a continuación, el intercambio espiritual del hombre que reza con Dios benevolente" 42 .

\section{En los limites de la fenomenología}

Llegados a este punto, nos gustaría manifestar algunas inquietudes que la fenomenología de la donación despierta, aunque sea desde luego posible que las preguntas no se ajusten con toda fidelidad a los planteos e intenciones de Marion. La primera inquietud es si la estrategia propuesta por el francés para erigir una filosofia no metafísica no implica acaso una puesta en jaque de su propia crítica a la onto-teología. Dicho de otro modo, nos preguntamos si la ontología gradual como modo de superar la filosofia onto-teológica no termina siendo una salida de la metafisica por la metafisica, esto es, si no termina siendo una estrategia de sustitución de un sistema por otro. En este sentido, también se podría cuestionar si un panteísmo fenoménico (como el que implica la gradación entre fenómenos comunes, fenómenos saturados y fenómenos doblemente saturados) no conlleva, a su vez, un desplazamiento de la regionalización del primer al tercer término de la jerarquía ontológica y, por tanto, si dicha jerarquía no se ve afectada por un posible regreso al infinito. Además, contrariamente a los intentos por destacar el carácter excepcional de los fenómenos saturados, Marion insiste al menos tres veces en el capítulo IV de Étant donné en su carácter paradigmático, contra el cual hay que medir por "defecto o simplificación todas las otras figuras de la automanifestación de la donación"43. Así, nuestro autor advierte: "Aunque paradojal, o por eso mismo, el fenómeno saturado no debe de ningún modo entenderse como un caso excepcional, es decir vagamente irracional (y, por qué no, teológico) de la fenomenalidad. Cumple al contrario el desarrollo coherente y racional de la definición más operatoria del fenómeno: solo él aparece verdaderamente como sí mismo, de sí mismo y a partir de sí mismo... Nombramos a esta aparición puramente de sí, a partir de sí, que no somete su posibilidad a ninguna determinación previa, una automanifestación. Se trata pura y simplemente del fenómeno tomado en su acepción plenaria,

390 en sintesis, de su figura normativa, en relación con la cual todas las otras se definen y se declinan eventualmente por defecto o simplificación"44.

\footnotetext{
42 Ibid. pp. 46-47.

43 Marion, J.-L., ED, pp. 358-359.

44 Ibid.
} 
Se abre aquí un segundo campo de problemas, pues afirmaciones como la recién citada reflejan una búsqueda por subordinar a la filosofia bajo la teología, lo cual, según sostiene Marion en su artículo de 2009 "Sobre el fundamento de la distinción entre teología y filosofia", inscribiría a la teología en el modelo propio de aquella filosofia que busca subordinar a las otras disciplinas, es decir, en el modelo de la metafísica que él critica: "Si estamos absolutamente obligados a enfatizar una diferencia [entre filosofia y teologia], debemos decir que la teología permite una donación más inmensa (precisamente porque es revelada, mediata y proveniente de un allende radical) que la de la filosofia y, por supuesto, que aquella de las ciencias regionales positivas... Este espacio... pone a la teología en una posición dominante en relación con todo otro conocimiento, en particular, la filosofia"45.

Más aun, para Marion no se trata solo de asignar un lugar privilegiado a la teología en virtud de su objeto más elevado o porque permite una donación más inmensa. Se trata incluso de darle una función a la teología en relación con una filosofia que se ha extraviado: la "extensión [del dominio de la racionalidad] de lo dado también constituye, para toda filosofia que desea superar algún día el 'fin de la metafísica' (si es que puede alguna vez), el desafío más serio, pero el único capaz de salvarla"46.

Ahora bien, Marion podría contra-argumentar que su planteo no debe entenderse en los términos de la filosofia metafisica onto-teológica que él se propone superar. Pero también es cierto que si bien todavia en Étant donné encontramos matices en el giro teológico (el fenómeno saturado no debe entenderse como "irracional", es decir, "teológico", sino como un fenómeno "coherente y racional"47), también lo es que el limite entre filosofia y teología parece desdibujarse a lo largo de la obra de Marion. En este sentido, nos preguntamos si aquel panteísmo fenoménico que se vislumbra a través de la jerarquía de fenómenos no implica una salida de la fenomenología. Dar lugar a la posibilidad

\footnotetext{
${ }^{45}$ La cita continúa: "la teología precede a la filosofia a su manera, en el camino de su propio ensanchamiento como una filosofia no-metafísica.... No le impone ninguna restricción [a la filosofia], sino que propone su liberación de los obstáculos que pesan sobre la constitución ontoteológica de la metafísica. Por lo tanto, la teología se convierte en la conciencia racional culposa de la filosofia, porque puede (y debe) considerar inmediatamente un hecho que es sin embargo mediato -y enteramente un hecho excepcional, es verdad- entre todos los demás hechos nuevos que la filosofia del 'fin de la metafisica' debe considerar y luchar tanto por integrar" (Marion, J.-L., "On the Foundation of the Distinction Between Theology and Philosophy", en: Budhi: A Journal of Ideas and Culture, v. XIII, 1-3 (2009), pp. 55-56).

${ }^{46}$ Ibid.

${ }^{47}$ Marion, J.-L., ED, p. 359.
} 
de la revelación (anti fenoménica por excelencia), ¿no implica una renuncia a la filosofia? ¿Cómo convive una filosofia fenomenológica con una teología de la revelación? ¿Se suceden, se superponen, se excluyen? Tal vez haya que entender la fenomenología de la donación más como una fansiología que como una fenomenología, esto es, más como un discurso sobre lo que es dado, se da o que aparece, que como una ciencia de los fenómenos y de lo fenoménico. A fin de cuentas, si el fenómeno saturado no es una excepción, se puede pensar que el fenómeno doblemente saturado es casi como la donación en estado cero, como el ser de todo ente, como aquello que permite que todo fenómeno aparezca, de modo que el de Marion sería el discurso no de lo que aparece en tanto que aparece, sino de lo que aparece en tanto que es dado -y dado por un alguien/eso/algo trascendente-48. En este sentido, habría que concluir que ambos, tanto el giro como desvío como el giro como retorno, pueden reducirse a un único sentido, ya que indican en una sola dirección: la trascendencia.

Por último, incluso si concedemos todo lo anterior, ¿es la posibilidad de la manifestación fenoménica de la trascendencia la única vía de renovación y ensanchamiento de la filosofia? En otras palabras, quizás la pregunta más importante sea por la necesidad de la alternativa teológica o, más específicamente, por la necesidad de la vía de la teología de la revelación como salida de la filosofia onto-teológica. Porque, ¿no implica el salto teológico un abandono del rigor pretendido de la fenomenología? ¿Por qué abandonar toda alternativa inmanente ${ }^{49}$ ? En la compilación de artículos intitulada Trascendencia y sobreabundancia. Fenomenología de la religión y filosofia primera, Luis Rabanaque señala, en su artículo "Dios en la fenomenología de Husserl"50, que los caminos a Dios pueden ser por vía filosófica y no solo por vía teológica. Sin embargo, incluso concediendo la vía teológica, podemos cuestionar el privilegio del cristianismo sobre otras religiones.

\footnotetext{
${ }^{48}$ En concordancia encontramos el epígrafe de Étant donné: "Axioma: no podemos saber nada por nosotros mismos, todo saber auténtico debe sernos dado" (Novalis, "Brouillon général", en: Schriften, Stuttgart: W. Kohlhammer, 1960, v. III, p. 441 (§902), citado en: Marion, J.-L., ED, p. 1). ¿Podriamos decir otro tanto para las acciones?

49 Según Alexander Schnell, en Qu'est-ce que le phénomène?, París: Vrin, 2014, cuando Husserl se encontró con aquel límite que se le adjudica como su límite último (la pregunta por la fenomenalidad o fenomenabilidad de la conciencia absoluta del tiempo como aquella pregunta que indica a las condiciones inaparentes y pasivas de lo aparente/apareciente y activo), no buscó salir de la filosofia para superar el límite, sino que dio un giro en su método fenomenológico desde la fenomenología genética hacia la fenomenología constructiva.

${ }^{50}$ Rabanaque, L., "Dios en la fenomenologia de Husserl", en: Scannone S.I., J.C., Walton, R. y J.P. Esperón (eds.), Trascendencia y sobreabundancia. Fenomenología de la religión y filosofia primera, Buenos Aires: Biblos, 2015, pp. 45-60.
} 
Por último, dejando de lado la modalidad religiosa del tránsito entre lo fenoménico y lo trascendente, queda por preguntar por qué este tránsito se propone en términos positivos y no negativos. De hecho, otros panteísmos, como el del Romanticismo de Jena, suelen ser ontologías negativas, y es justamente la apofántica la que permite un vínculo, aunque inefable, con lo trascendente. La posibilidad de la manifestación de lo infinito en lo finito es la esencia de la estética de esa generación, pero dicha manifestación no aparece en la forma determinada de un contacto religioso, según las previsiones de un texto, sino que está abierta a la espontaneidad. Es, en efecto, espontaneidad y arbitrariedad, instantaneidad e intuición. La materialización se da bajo la figura de una obra de arte: lo infinito se materializa o informa gracias al arte del genio y a su intuición estética ${ }^{51}$. A nuestro entender, la posibilidad de la revelación no significa otra cosa que la posibilidad de la intuición intelectual (en palabras de Marion, intuitio vaga), es decir, de llegar a lo absoluto; como dirá Hegel de Schelling al comienzo de su Ciencia de la lógica: de un pistoletazo ${ }^{52}$. Por lo tanto, si para Marion la limitación de la donación en Kant y Husserl se debe a cierto "temor... a que reviva la causa de Dios" ${ }^{53}$, ¿no podríamos pensar al revés: que la necesidad de nombrar la manifestación del absoluto bajo la forma de una revelación de Dios en Jesucristo como su encarnación es un recubrimiento o enmascaramiento (como diría Nietzsche) del ser por temor al abismo o, directamente, un recubrimiento del "abismo" -de ese mismo abismo ante el cual, según Marion, Husserl retrocede encerrándolo con el concepto-?

Siguiendo las metáforas jurídicas y geográficas de Kant, tal vez podamos concluir que la diferencia entre la teología y la filosofia no radica en una diferencia de territorios, sino en las maneras de conquistar dichos territorios y en los modos de ser ciudadanos en $\operatorname{ellos}^{54}$. No se trata de que la filosofia no deba o no pueda conquistar el territorio que la teología de la revelación parece adjudicarse, pero la teología parece extender sus pretensiones más allá de lo

\footnotetext{
51 Sostiene esto, por ejemplo, Schelling en su Sistema del idealismo trascendental.

52 Es verdad que no asume la intuición intelectual, sino de apertura a la donación, pero también llega a hablar de intuición libre: "la intuición despliega un aumento que el concepto no puede ordenar, y que por tanto la intención no puede prever; de manera que la intuición ya no se encuentra ligada a la intención, sino que se libera de ella, se erige en una intuición libre (intuitio vaga)" (Marion, J.-L., ED, p. 370).

53 Ibid., p. 383.

54 En este sentido, podemos releer la metáfora de la isla (según la cual solo hay un territorio firme -el de la isla-, tras cuyos limites hay un mar tempestuoso en el que uno puede aventurarse pero no como en un territorio firme ( $c f$. Kant, I., Crítica de la razón pura, México D.F.: Porrúa, 1982, p. 142), para remarcar que en realidad entre la isla y el mar hay una continuidad territorial, no pertenecen a mundos inconexos.
} 
fenomenológico. En este punto, la crítica de Janicaud no ha sido respondida por Marion, quien ha, por el contrario, reforzado su respuesta desde la postura teológica. Al menos en la disputa por la propiedad del territorio del allende, Marion se autoerige como juez y resuelve la disputa por remisión a sus objetos: dado que el objeto de la teología es el supremo, Dios, entonces la teología es la propietaria de ese territorio. Mientras que, en realidad, la filosofia no pretende disponer con exclusividad de territorios que no son firmes, que incluso quizás no son más que un espejismo, pero que le quedan dentro de su horizonte de posibilidades (y no, como pretende Marion, en el reino de lo imposible). ¿No es virtud de la filosofia la capacidad de explorar esos límites, ese territorio abismal, sin fin y sin fondo, soportando una mirada sostenida en lo aparentemente insupportable, invisable, irregardable? Así como en la ilusión trascendental, la pretensión de incorporar lo allende a los límites de la racionalidad es inevitable ${ }^{55}$, pero una ensoñación en la que no debemos caer por largo tiempo. Como Ulises, la filosofia navega entre Escila y Caribdis, entre la fe y el escepticismo. Su potencia reside en convertir en su morada -expulsiva y acogedora a la veza ese abismo que se abre entre ambas amenazas. La filosofia nos invita a no perder la perspectiva y a no renunciar al rigor que exige transitar el abismo, incluso si, como Ulises, decidimos de tanto en tanto ir atados al mástil para dejarnos seducir, para entregarnos a la Schwärmerei del allende.

Recibido: $11 / 05 / 2017$

Aceptado: 17/09/2018

\section{Bibliografia}

Husserl, E., Idées directrices pour une phénoménologie et une philosophie phénoménologique pures. Tome premier Introduction générale à la phénoménologie pure, Ricœur, P. (trad.), Paris: Gallimard, 1950.

Husserl, E., Ideas relativas a una fenomenología pura y una filosofia fenomenológica, Gaos, J. (trad.), México D.F./Buenos Aires: FCE, 1962.

Husserl, E., Phantasia, conscience d'image, souvenir, Kassis, R. y J.-F. Pestureau (trads.), París: Jérôme Millon, 2002.

Janicaud, D., "Le tournant théologique de la phénoménologie française", en: La phénomenologie dans tous ses états, París: Gallimard, 2009.

Kant, I., Crítica de la razón pura, García Morente, M. (trad.), , México D.F.: Porrúa, 1982.

\footnotetext{
${ }^{55} \mathrm{El}$ problema con la ilusión trascendental es que "aun después de descubierto su espejismo, no cesa, sin embargo, de engañar y de empujas la razón, sin descanso, a momentáneos errores, que necesitan de continuo ser remediados" (ibid., pp. 167-168).
} 
Kant, I., Critica del discernimiento, García Morente, M. (trad.), México D.F: Porrúa, 1985. Marion, J.-L., "El cruce lo visible y lo invisible", en: El cruce de lo visible, Bassas, J. y J. Masó (trads.), Castellón: Ellago Ediciones, 2006.

Marion, J.-L., Au lieu de soi. L'approche de saint Augustin, París: PUF, 2008.

Marion, J.-L., "On the Foundation of the Distinction Between Theology and Philosophy", en: Budhi: A Journal of Ideas and Culture, v. XIII, 1-3 (2009), pp. 55-56.

Marion, J.-L., La rigueur des choses. Entretiens avec Dan Arbib, París: Flammarion, 2012. Marion, J.-L., Étant donné, París: PUF, 2013.

Marion, J.-L., Réduction et donation, París: PUF, 2015.

Novalis, "Brouillon général", en: Schriften, Stuttgart: W. Kohlhammer, 1960.

Rabanaque, L.R., "Dios en la fenomenologia de Husserl”, en: Scannone S.I., J. C., Walton, R., y J.P Esperón (eds.), Trascendencia y sobreabundancia. Fenomenología de la religión y filosofia primera, Buenos Aires: Biblos, 2015, pp. 45-60.

Ricœur, P., "Sur la phénoménologie", en: Á l'école de la phénomenologie, París: Vrin, 1986.

Schelling, F.W.J., Sistema del idealismo trascendental, Rivera de Rosales, J. y V. López Dominguez (trads.), Barcelona: Anthropos, 2005.

Schnell, A., Qu'est-ce que le phénomène?, París: Vrin, 2014.

Walton, R., "Reducción fenomenológica y figuras de la excedencia", en: Tópicos, 16 (2008), pp. 169-187. 